\title{
FIRST REPORT ON BIODEGRADATION OF LOW DENSITY POLYETHYLENE BY RICE MOTH LARVAE, Corcyra cephalonica (STAINTON)
}

\author{
Sheetal Suresh Kesti", Shivasharana Chandrabanda Thimmappa* \\ * Karnatak University, Department of Studies in Biotechnology and Microbiology, Dharwad, Karnataka, \\ India \\ corresponding author: Shivasharana Chandrabanda Thimmappa, e-mail: shivaskud@gmail.com
}

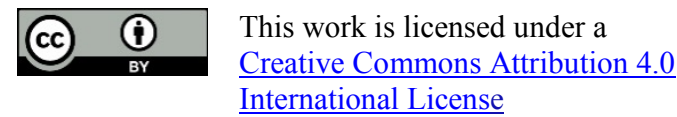

\author{
Original scientific paper \\ Received: August $12^{\text {th }}, 2019$ \\ Accepted: September 13 ${ }^{\text {th }}, 2019$ \\ HAE-1918 \\ https://doi.org/10.33765/thate.9.4.2
}

\begin{abstract}
Corcyra cephalonica (Stainton), rice moth larvae were used for biodegradation of low density polyethylene (LDPE). The role of larval gut microorganisms in LDPE biodegradation was studied by force feeding the larvae with a cocktail of antibiotic solution. After force feeding, the larvae were left in contact with LDPE films. Larvae which were unfed with antibiotic solution were used as controls. Degradation rate was checked by measuring the weight loss after 20 day period of contact with LDPE films in insect rearing bottles. The DNA of both antibiotic fed and unfed larvae was isolated. There was no DNA band of antibiotic fed larvae which indicated the gut microorganisms were eliminated. Larvae fed with antibiotic digested $21 \%$ and those which were unfed with antibiotic showed $25 \%$ weight loss of LDPE films. We report the gut microorganisms were not solely responsible for biodegradation of LDPE. The digestive enzymes could also play a major role in digesting the plastic in insect's gut.
\end{abstract}

Keywords: Corcyra cephalonica, rice moth larvae, low density polyethylene, antibiotic

\section{INTRODUCTION}

The rice moth, Corcyra cephalonica (Stainton) is a stored grain pest distributed all over Asia, Africa, Europe and North America [1]. It is a serious pest of rice and also feeds on other grains such as wheat, maize, groundnut, sorghum and millets [2]. Corcyra cephalonica is mostly used for mass production of biological agents and entomopathogenic nematodes [3].
Biodegradation of plastics by free living microorganisms have been reported till now but the efficiency of degradation was very low compared to the degradation rate by using insects and their gut microorganisms [4].

Biodegradation of plastics by using Indian meal worms, Plodia interpunctella was first reported by Yang et al. (2014). According to Yang et al. the gut microorganisms played a major role in polyethylene degradation. Enterobacter asburiae YT1 and Bacillus sp. 
YP1 were capable of degrading PE in Indian meal worm's gut [4]. Polystyrene (PS) biodegradation by yellow meal worms, Tenebrio molitor was studied by Yang et al. (2015) and reported the mass loss of $47.7 \%$ of PS over a 16 day test period [5]. Further the role of gut microorganisms and the effects on life cycle of yellow meal worms when fed with PS was also studied [6]. Biodegradation of Polyethylene (PE) by Greater wax moth larvae, Galleria mellonella was studied by Bombelli et al. (2017) wherein approximately 100 larvae consumed $92 \mathrm{mg}$ of PE in 2 hours [7]. Hyun Gi Kong (2019) studied a detail mechanism of PE degradation in greater wax moth larvae. According to the study, gut microorganisms where not solely responsible for PE digestion in larval gut. Elimination of gut microorganisms by force feeding the larvae with a cocktail of antibiotics revealed that the larvae in the absence of gut microorganisms digested $\mathrm{PE}$ and was confirmed by Fourier Transform Infrared Spectroscopy (FTIR) and Gas-chromatography (GC) [8].

The present work aims to study the role of Corcyra cephalonica larvae and the gut microorganisms in LDPE degradation. Insects were reared on an artificial medium at $28 \pm 1$ ${ }^{\circ} \mathrm{C}$, a relative humidity of $60 \pm 5 \%$ and $12 \mathrm{~L}$ : $12 \mathrm{D}$ during the study period [9]. Among 50 larvae used for the study, 25 larvae were force fed with $9 \mu 1$ of $1 \mathrm{mg} / \mathrm{mL}$ antibiotic mixtures (ampicillin, kanamycin and neomycin) prepared in sterile water and the remaining 25 larvae were used as controls. Both antibiotic fed and unfed larvae were left in contact with LDPE films cut in required sizes in two separate insect rearing bottles. The weight loss of LDPE was measured after 20 days and found that there was a gradual loss of LDPE weight as the larvae consumed the films. Interestingly, we found that even in the absence of gut microorganisms i.e. the larvae which were fed with antibiotic, consumed nearly equal amount of LDPE as compared with the controls.

\section{EXPERIMENTAL}

\section{Materials}

Low Density polyethylene (LDPE) bags were purchased from the local market of Dharwad, Karnataka, India. The bags were wiped with 1 $\%$ Sodium dodecyl sulfate and $95 \%$ ethanol to remove the dust particles adhered to the bags.

\section{Rearing of insects in laboratory}

Corcyra cephalonica eggs (1cc) were procured from National Bureau of Agricultural Insect Resources (NBAIR), Bengaluru. The eggs were transferred into artificial medium which contained $2.5 \mathrm{~kg}$ of broken bajra, $75 \mathrm{~g}$ of groundnut seed powder, $5 \mathrm{~g}$ of yeast powder and $0.7 \mathrm{~g}$ of streptomycin sulphate. The growth of larvae was monitored regularly and the pupae were transferred to new chamber. The adults were transferred to an egg laying chamber supplemented with cotton swabs soaked in $5 \%$ honey. The eggs were again transferred to a new rearing chamber with artificial medium.

\section{The role of gut microorganisms in LDPE degradation}

The rice moth larvae, Corcyra cephalonica (Stainton) of $3^{\text {rd }}$ instar were selected for the study. Totally 50 larvae were collected from the rearing chamber and starved for 48 hours. 25 larvae were transferred to a bottle containing accurately weighed LDPE films of $5 \mathrm{~cm}^{2}$ sizes and the remaining 25 larvae were force fed with a cocktail of antibiotic solution. Ampicillin, kanamycin and neomycin of 1 $\mathrm{mg} / \mathrm{mL}$ were prepared in sterile water and $9 \mu \mathrm{l}$ of mixture was force fed to larvae. Briefly, a Durham's tube was cut into small size of $2 \mathrm{~cm}$ and $3 \mu \mathrm{l}$ of each antibiotic solution was added to it. Force feeding of antibiotic was performed by holding the head of the larva into the Durham's tube for 15 seconds (Figure 1). The larvae after force feeding with antibiotic solution were transferred to a bottle 
containing accurately weighed LDPE films of $5 \mathrm{~cm}^{2}$ sizes.

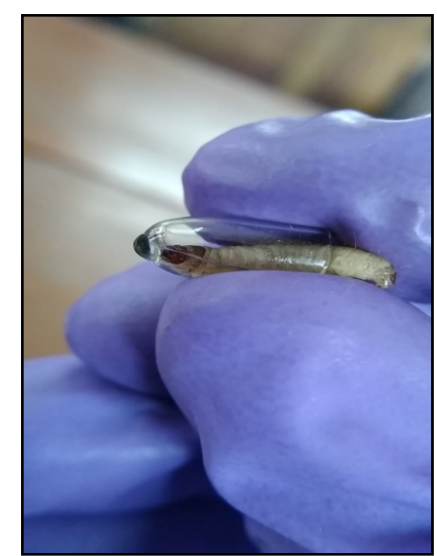

Figure 1. Force feeding of antibiotic ( $9 \mu 1$ of 1 $\mathrm{mg} / \mathrm{mL}$ ) solution to larvae

\section{Isolation and culture of gut microorganisms (Culture dependent method)}

Five larvae from each group were used for isolation of guts. The guts were isolated as described by Yang et al. (2014) with slight modifications. The larvae were surface sterilized with $70 \%$ ethanol and sterile water. The guts were drawn out and added to $1.5 \mathrm{ml}$ tubes containing $500 \mu \mathrm{l}$ of sterile saline water $(0.9 \%)$. The tubes were vortexed briefly and the gut tissues were removed carefully with the help of sterile pipette and the suspension was used as an inoculum. $200 \mu 1$ of the suspension from both the tubes was spread on nutrient agar (NA) plates and incubated at 37 ${ }^{\circ} \mathrm{C}$ for 24 hours [4].

\section{Isolation of gut microbial DNA (Culture independent method)}

Isolation of gut microbial DNA was performed as described by Kong et al. (2019) with slight modifications [8]. Briefly, single larva from both the groups was collected and the guts were drawn out. The guts were homogenized in $1.5 \mathrm{ml}$ microcentrifuge tubes containing 400 $\mu \mathrm{l}$ of TE buffer (Tris $10 \mathrm{mM}$, EDTA $1 \mathrm{mM}$, $\mathrm{pH}$ 8.0) and sonicated (30 Amplitude, $1 \mathrm{~s}$ pulse). $20 \mu \mathrm{g} / \mathrm{mL}$ RNase, $0.5 \%$ sodium dodecyl sulphate (SDS) and $10 \mu \mathrm{l}$ of 100 $\mu \mathrm{g} / \mathrm{ml}$ proteinase $\mathrm{K}$ was added to the tubes and incubated at $56{ }^{\circ} \mathrm{C}$ for 2 hours. An equal volume of phenol : chloroform : isoamylalcohol $(25: 24: 1)$ was added to all the tubes and centrifuged at $10000 \mathrm{rpm}$ for 15 minutes. A clear supernatant was transferred to a new $1.5 \mathrm{ml}$ tube and extracted with an equal volume of Chloform : Isoamylalcohol (24:1). The tubes were vortexed briefly and centrifuged at $10000 \mathrm{rpm}$ for 15 minutes. Supernatant was transferred to new $1.5 \mathrm{ml}$ tube and DNA was precipitated by adding 0.6 volume chilled isopropanol. No DNA was precipitated in the larvae fed with antibiotic solution. The tubes were centrifuged at 10000 rpm for 5 minutes and supernatant was discarded. The DNA pellet from the antibiotic unfed larvae was washed with $70 \%$ ethanol. The tube was centrifuged at $10000 \mathrm{rpm}$ for 5 minutes and the pellet was dried and dissolved in $50 \mu \mathrm{l}$ of sterile TE buffer and stored at $4{ }^{\circ} \mathrm{C}$ [8].

\section{RESULTS AND DISCUSSION}

\section{Weight loss of LPDE films}

The LDPE films from both the rearing bottles were collected after 20 days, wiped with $95 \%$ ethanol and weighed accurately to determine the weight loss. Weight loss was calculated by using the following formula:

\section{Weight loss $(\%)=\{($ Initial weight - Final weight $/$ Initial weight $\} \cdot 100$}

Table 1 indicates the percentage of weight loss of LDPE films. The larvae force fed with antibiotic solution to eliminate the gut microorganisms showed $21 \%$ of weight loss and the larvae which were not fed with antibiotic solution showed $25 \%$. Figure 2 represents the holes made by rice moth larvae on LDPE films during experimentation. Yang et al. (2015) carried out the similar experiments on Polystyrene (Styrofoam) by using Tenebrio molitor, a yellow meal worms and found $47.7 \%$ of Styrofoam ingested by worms in a 16 day test period [5]. In a work 
conducted by Bombelli et al. (2017), a total of 100 Greater wax moth larvae (Galleria mellonella), consumed $92 \mathrm{mg}$ of polyethylene in a 2 hour period [7].

Table 1. Percentage of weight loss of LDPE films

\begin{tabular}{|c|c|c|c|}
\hline & $\begin{array}{c}\text { Initial } \\
\text { weight } \\
(\mathrm{g})\end{array}$ & $\begin{array}{c}\text { Final } \\
\text { weight } \\
(\mathrm{g})\end{array}$ & $\begin{array}{c}\text { Weight } \\
\text { loss } \\
(\%)\end{array}$ \\
\hline $\begin{array}{c}\text { LDPE films } \\
\text { from } \\
\text { antibiotic } \\
\text { unfed larvae } \\
\text { bottle }\end{array}$ & 1.0 & 0.75 & 25 \\
\hline $\begin{array}{c}\text { LDPE films } \\
\text { from } \\
\text { antibiotic fed } \\
\text { larvae bottle }\end{array}$ & 1.0 & 0.79 & 21 \\
\hline
\end{tabular}

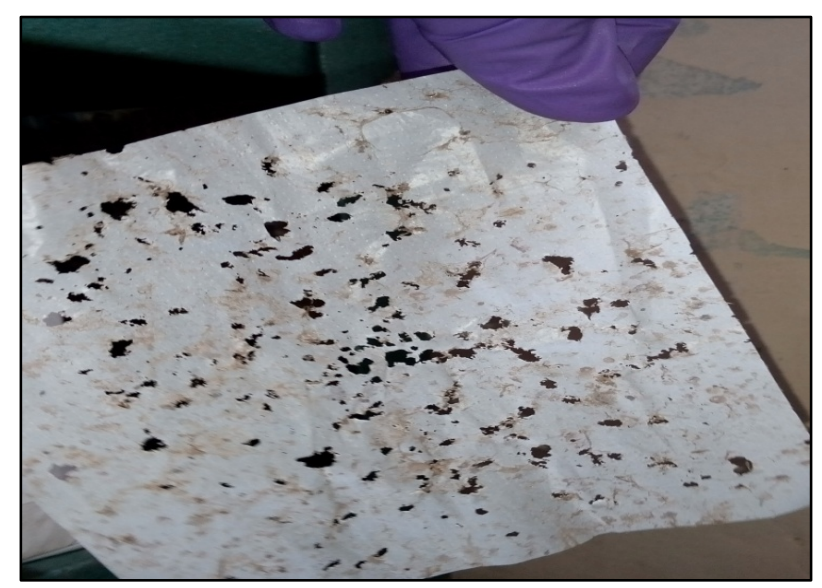

Figure 2. Appearance of holes on LDPE films made by Rice moth larvae

\section{Culture dependent method to identify the role of gut microorganisms}

The larvae which were force fed with antibiotic solution showed no growth, whereas those which were not fed with antibiotic solution showed the growth of bacterial colonies on NA plates as shown in Figure 3. Ampicillin was used to eliminate Gram negative bacteria, kanamycin for Gram positive bacteria and neomycin for the elimination of yeast from the gut of the larvae. Kong et al. (2019) reported the similar studies on Greater wax moth, Galleria mellonella larvae to know the role of gut microorganisms in polyethylene degradation [8].

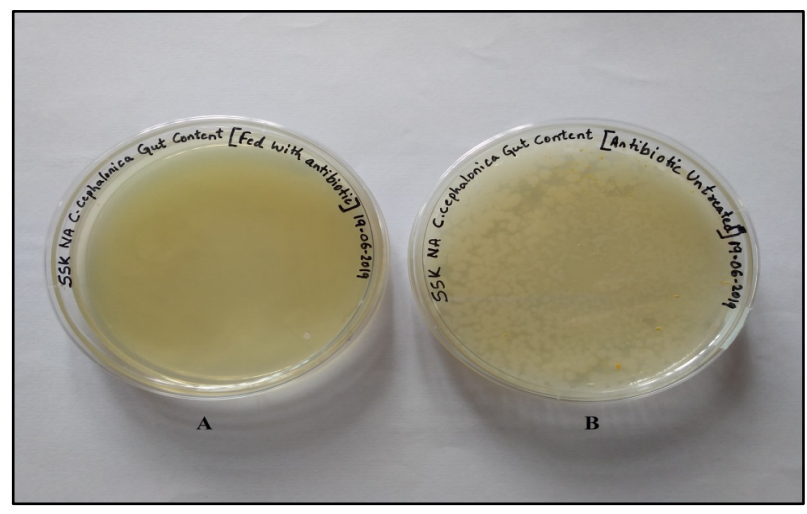

Figure 3. Nutrient agar plates: Antibiotic fed larval gut content (A) and Antibiotic unfed larval gut content $(\mathrm{B})$

\section{Larval gut microbial DNA}

There was no DNA in antibiotic fed larvae. However, both the elutions were run on $0.8 \%$ agarose gel. The larvae fed with antibiotic showed no DNA band whereas those which were not fed with antibiotic showed the DNA band (Figure 4). Studies conducted by Kong et al. (2019) on Greater wax moth larvae indicated that the gut microorganisms were not solely responsible for polyethylene and beeswax degradation [8].

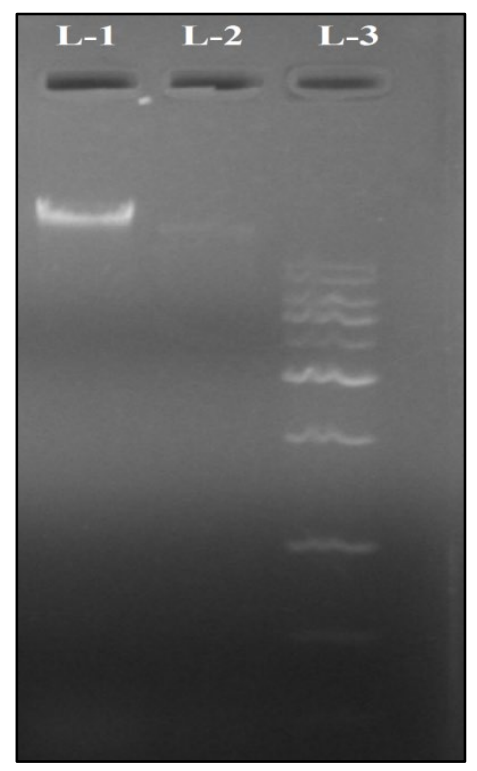

Figure 4. Lane 1- Gut microbial DNA of larvae unfed with antibiotic, lane 2- larvae fed with antibiotic and lane 3 - DNA ladder $1 \mathrm{~kb}$ 


\section{CONCLUSION}

The aim of this study was to identify the role of gut microorganisms of Corcyra cephalonica larvae in LDPE biodegradation in their guts. Weight loss method indicated that the gut microorganisms are not solely responsible for LDPE digestion in insect's gut. An average of $23 \%$ weight loss of LDPE was observed in both antibiotic fed and untreated larvae. The gut content of the larvae which were force fed with antibiotic and spread on NA plates showed no colonies and those which were not fed with antibiotic showed bacterial colonies after 24 hours of incubation. This indicated that the gut microorganisms were susceptible to antibiotic solution. The DNA bands were not observed in the larvae which were fed with antibiotic solution. This indicated that the larvae were able to function and digest LDPE with little or no role of gut microorganisms. However, this is the first report on biodegradation of low density polyethylene by rice moth larvae. The future work aims to study the mechanisms involved in the gut of rice moth larva in degrading the plastic.

\section{REFERENCES}

[1] A.S. Atwal, G.S. Dhaliwal, Agricultural Pests of South Asia and Their Management, Kalayani Publishers, New Delhi, India, 2008.

[2] M.A. Malek, B. Parveen, Effect of insect infestation on the weight loss and viability of stored BR 3 paddy, Bangladesh Journal of Zoology 78(1989) 7, 796 .

[3] B. Gopal, B.T. Resham, T. Sundar, S.B. Ghana, Reproductive biology study of Corcyra cephalonica (Stainton) under laboratory condition in Chitwan, Nepal, Journal of Innovative Biology 1(2014) 4, 206-209.

[4] J. Yang, Y. Yang, W. Wu, J. Zhao, L. Jiang, Evidence of polyethylene biodegradation by bacterial strains from the guts of plastic-eating waxworms,
Environmental Science and Technology 48(2014), 13776-13784.

[5] Y. Yang, J. Yang, W. Wu, J. Zhao, Y. Song, L. Gao, R. Yang and L. Jiang, Biodegradation and Mineralization of Polystyrene by Plastic-Eating Mealworms: Part 1. Chemical and Physical Characterization and Isotopic Tests, Environmental Science and Technology 49(2015), 12080-12086.

[6] S.S. Yang et al., Biodegradation of Polystyrene wastes in Yellow Mealworms (larvae of Tenebrio molitor Linnaeus): $\quad$ Factors affecting Biodegradation rates and the ability of Polystyrene fed-larvae to complete their life cycle, Chemosphere 191(2018), 979989.

[7] P. Bombelli, C.J. Howe, F. Bertocchini, Polyethylene bio-degradation by the caterpillars of wax moth Galleria mellonella, Current Biology 27(2017), R283-R293.

[8] H.G. Kong, H.H. Kim, J. Chung, J. Jun, S. Lee, H. Kim, S. Jeon, S.G. Park, J. Bhak, C. Rui, The Galleria mellonella Hologenome Supports MicrobiotaIndependent Metabolism of Long chain Hydrocarbon Beeswax, Cell Reports 26(2019), 2451-2464.

[9] I. Ara, M.A. Haque, M.N. Bari, N. Ahmed, N. Islam, Development of rice meal moth, Corcyra cephalonica (Stainton) on different food media, University Journal of Zoology Rajshahi University 24(2005), 17-21.

\section{Acknowledgements}

Authors are grateful to the Department of Studies in Biotechnology and Microbiology, Karnatak University Dharwad for providing laboratory facilities and National Bureau of Agricultural Insect Resources (NBAIR), Bengaluru, for providing insect cultures (National Accession number: Corcyra cephalonica - NBAII-MP-CYR-01). 